\title{
An adaptive mesh method for time dependent singularly perturbed differential-difference equations
}

https://doi.org/10.1515/nleng-2018-0075

Received May 6, 2018; accepted May 10, 2018.

\begin{abstract}
In this paper, a time dependent singularly perturbed differential-difference convection-diffusion equation is solved numerically by using an adaptive grid method. Similar boundary value problems arise in computational neuroscience in determination of the behaviour of a neuron to random synaptic inputs. The mesh is constructed adaptively by using the concept of entorpy function. In the proposed scheme, prior information of the width and position of the layers are not required. The method is independent of perturbation parameter $\varepsilon$ and gives us an oscillation free solution, without any user introduced parameters. Numerical examples are presented to show the accuracy and efficiency of the proposed scheme.
\end{abstract}

Keywords: Differential-difference equations, Singular perturbation, Boundary layer, Entropy, adaptive mesh, Central finite difference scheme

MSC: 65L11, 65M12, 35K20

\section{Introduction}

Singularly perturbed partial differential equations arise in a wide variety of application fields such as biosciences, economics, material science, medicine, robotics etc. $[5,14$, $20]$ and in the last few decades there has been a growing interest in the study of delay differential equations [3, 4, 9]. In 1965 Stein [19] proposed a mathematical model of neuronal variability to study the stochastic movement of neuron. Later he generalized the model to study the distribu-

\footnotetext{
*Corresponding Author: P. Pramod Chakravarthy, Department of Mathematics, Visvesvaraya National Institute of Technology, Nagpur, 440010, India, E-Mail: pramodpodila@yahoo.co.in

Kamalesh Kumar, Department of Mathematics, Visvesvaraya National Institute of Technology, Nagpur, 440010, India, E-Mail: kamleshjangir2012@gmail.com
}

tion of past synaptic potential amplitudes. In 1991 Musila and Lansky [13] generalised the Stein's model and given the following mathematical model:

$$
\begin{aligned}
& -\frac{\partial u}{\partial t}=\frac{\sigma^{2}}{2} \frac{\partial^{2} u}{\partial x^{2}}+\left(\mu_{D}-\frac{x}{\tau}\right) \frac{\partial u}{\partial x}+\lambda_{s} u\left(x+a_{s}, t\right) \\
& +\omega_{s} u\left(x+i_{s}, t\right)-\left(\lambda_{s}+\omega_{s}\right) u(x, t) .
\end{aligned}
$$

Here, due to exponential decay between two consecutive jumps caused by the input processes, the first derivative term will occur. The membrane potential decays exponentially to the resting level with a membrane time constant $\tau$ and $\mu_{D}$ and $\sigma$ are diffusion moments of Wiener process characterizing the influence of the dentritic synapses on the cell excitability. The reaction terms correspond to the superposition of excitatory and inhibitory inputs and we can assume that they are Poissonian. The excitatory input contributes to the membrane potential by amplitude $a_{s}$ with intensity $\lambda_{s}$ and similarly the inhibitory input contributes by amplitude $i_{s}$ with intensity $\omega_{s}$. This model makes available time evolution of the trajectories of the membrane potential.

Lange and Miura [11], studied the asymptotic analysis of singularly perturbed boundary value problems for differential-difference equations in ordinary differential equations. This study motivated many researchers to work on numerics of singularly perturbed differential-difference equations in ordinary differential equations and partial differential equations. Here we are concerned with the partial differential equations of convection-diffusion type with general shift arguments, which are singularly perturbed. It is noticeable that the behavior of the solution of singularly perturbed partial differential equations with shift arguments are essentially different from those of without shift arguments.

Ramesh and Kadalbajoo [15] proposed a numerical scheme based on classical finite differences on the Shishkin mesh for solving singularly perturbed partial differential-difference equations with small shift arguments. The method is found to be uniformly convergent with respect to the perturbation parameter. Kumar and Kadalbajoo [9] had given a B-spline collocation method on fitted mesh for solving singularly perturbed partial 
differential-difference equations with small shift arguments. In $[9,15]$, they approximated the shifted terms by Taylor series and difference schemes are applied. Such methods will work when shifts are smaller than perturbation parameter and fails in the case when shifts are bigger than perturbation parameter. Bansal et. al.[3, 4] developed parameter uniform numerical schemes to find the approximate solution of time dependent singularly perturbed convection-diffusion-reaction problems with general shift arguments in space variable, which work for small shifts as well as large shifts. Rao and Chakravarthy [16] had given a fitted numerical scheme for singularly perturbed one-dimensional parabolic partial differential equations with small shifts.

If we solve singularly perturbed partial differential equations using central finite difference scheme on a uniform mesh, it gives oscillatory solution, which shows that method is unstable. To deal with such situation, more mesh points in boundary layer region is required. To cater the need, layer adaptive meshes have been developed by Bakhvalov [2], Gartland [8] and others. A special piecewise uniform meshes have been proposed by Miller and Shishkin [12]. Shishkin meshes are used widely because of their simplicity. The major drawback of Shishkin meshes is the requirement of prior information of the location of the layer regions. To overcome this drawback, in this paper, we proposed an adaptive mesh method using the concept of entropy function for solving convection-diffusionreaction singularly perturbed delay parabolic partial differential equations. The method is independent of perturbation parameter $\varepsilon$ and gives us an oscillation free solution, without any user introduced parameters.

The paper is organized as follows: The problem under consideration is stated and the sufficient compatibility conditions on the initial and boundary data to guarantee the existence, uniqueness and appropriate regularity of the solutions to the problem are presented in Section 2. Numerical scheme and variable mesh algorithm is presented in Section 3. Section 4 is devoted to the stability and error analysis. To demonstrate the efficiency and applicability of the proposed numerical scheme, numerical experiments are carried out for three test problems and results are given in Section 5. The paper ends with conclusions in last section.

\section{Statement of the problem}

Let $\Omega=(0,1), D=\Omega \times(0, T]$, for some fixed time T and $\Gamma=$ $\Gamma_{l} \cup \Gamma_{b} \cup \Gamma_{r}$, where $\Gamma_{l}=\{(x, t):-\delta \leq x \leq 0$, and $0 \leq t \leq T\}$ and $\Gamma_{r}=\{(x, t): 1 \leq x \leq 1+\eta$, and $0 \leq t \leq T\}$ are the left and the right sides of the domain $\mathrm{D}$ and $\Gamma_{b}=[0,1]$.

In this paper, we consider a class of time dependent singularly perturbed partial differential difference equation with initial condition and the interval boundary conditions of the from:

$$
\begin{aligned}
& \frac{\partial u}{\partial t}-\varepsilon \frac{\partial^{2} u}{\partial x^{2}}+a(x) \frac{\partial u}{\partial x}+b(x) u(x, t)+c(x) u(x-\delta, t) \\
& +d(x) u(x+\eta, t)=f(x, t)
\end{aligned}
$$

where $(x, t) \in D$,

$\left\{\begin{array}{l}u(x, 0)=\phi_{b}(x), \quad \forall x \in \Gamma_{b}=[0,1], \\ u(x, t)=\phi_{l}(x, t), \\ \forall(x, t) \in \Gamma_{l}=\{(x, t):-\delta \leq x \leq 0, \text { and } 0 \leq t \leq T\}, \\ u(x, t)=\phi_{r}(x, t), \\ \forall(x, t) \in \Gamma_{r}=\{(x, t): 1 \leq x \leq 1+\eta, \text { and } 0 \leq t \leq T\},\end{array}\right.$

where $0<\varepsilon \ll 1$ is the singular perturbation parameter, $\delta, \eta>0$ represent the shift parameters. The functions $a(x), b(x), c(x), d(x), f(x, t), \phi_{b}(x), \phi_{l}(x, t), \phi_{r}(x, t)$ are assumed to be smooth and bounded functions on $\bar{D}$, that satisfy the conditions $b(x)+c(x)+d(x) \geq \alpha>0$ on $\Gamma_{b}=[0,1]$, for some constant $\alpha$. When $\delta=\eta=0$, the above problem reduces to singularly perturbed partial differential equation. If $a(x) \geq \beta>0$, for some constant $\beta$, $c(x)<0$ and $d(x)<0$ for all $x \in \Gamma_{b}=[0,1]$, then the solution exhibits boundary layer along $x=1$.

The problem (1) with initial condition and the interval boundary conditions (2), can be rewritten as

$$
\mathcal{L}_{\varepsilon} u(x, t)=F(x, t),
$$

where

$$
\mathcal{L}_{\varepsilon} u(x, t) \equiv\left\{\begin{array}{c}
\frac{\partial u}{\partial t}-\varepsilon \frac{\partial^{2} u}{\partial x^{2}}+a(x) \frac{\partial u}{\partial x}+b(x) u(x, t) \\
+d(x) u(x+\eta, t), \\
\text { if } 0<x \leq \delta, 0<t \leq T, \\
\frac{\partial u}{\partial t}-\varepsilon \frac{\partial^{2} u}{\partial x^{2}}+a(x) \frac{\partial u}{\partial x}+b(x) u(x, t) \\
+c(x) u(x-\delta, t)+d(x) u(x+\eta, t), \\
\text { if } \delta<x<1-\eta, 0<t \leq T, \\
\frac{\partial u}{\partial t}-\varepsilon \frac{\partial^{2} u}{\partial x^{2}}+a(x) \frac{\partial u}{\partial x}+b(x) u(x, t) \\
+c(x) u(x-\delta, t), \\
\text { if } 1-\eta \leq x \leq 1,0<t \leq T,
\end{array}\right.
$$

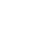


and

$$
F(x, t)=\left\{\begin{array}{c}
f(x, t)-c(x) \phi_{l}(x-\delta, t), \\
\text { if } 0<x \leq \delta, 0<t \leq T, \\
f(x, t), \\
\text { if } \delta<x<1-\eta, 0<t \leq T, \\
f(x, t)-d(x) \phi_{r}(x+\eta, t), \\
\text { if } 1-\eta \leq x \leq 1,0<t \leq T .
\end{array}\right.
$$

The existence and uniqueness of a solution of (1) can be established under the assumption that the data are Holder continuous and also satisfy appropriate compatibility conditions at the corner points $(0,0),(1,0),(-\delta, 0)$, $(1+\eta, 0)$ then the required compatibility conditions are[18]

$$
\begin{aligned}
& \phi_{b}(0)=\phi_{l}(0,0), \\
& \phi_{b}(1)=\phi_{r}(1,0),
\end{aligned}
$$

and

$$
\begin{aligned}
& \frac{\partial \phi_{l}(0,0)}{\partial t}-\varepsilon \frac{\partial^{2} \phi_{b}(0)}{\partial x^{2}}+a(0) \frac{\partial \phi_{b}(0)}{\partial x}+b(0) \phi_{b}(0) \\
& +c(0) \phi_{l}(-\delta, 0)+d(0) \phi_{b}(\eta)=f(0,0), \\
& \frac{\partial \phi_{r}(1,0)}{\partial t}-\varepsilon \frac{\partial^{2} \phi_{b}(1)}{\partial x^{2}}+a(1) \frac{\partial \phi_{b}(0)}{\partial x}+b(1) \phi_{b}(1) \\
& +c(1) \phi_{b}(1-\delta)+d(1) \phi_{r}(1+\eta, 0)=f(1,0) .
\end{aligned}
$$

These conditions guarantee that there exists a constant $C$ independent of $\varepsilon$ such that $\forall(x, t) \in \bar{D}$,

$$
|u(x, t)-u(x, 0)|=\left|u(x, t)-\phi_{b}(x)\right| \leq C t,
$$

and $|u(x, t)-u(0, t)|=\left|u(x, t)-\phi_{l}(0, t)\right| \leq C(1-x)$.

Lemma 2.1. (Maximum principle) Let the function $\varphi(x, t) \in C^{2,1}(\bar{D})$, such that $\mathcal{L}_{\varepsilon} \varphi(x, t) \geq 0$ in $D$ and $\varphi(x, t) \geq 0$ for all $(x, t) \in(\bar{D}-D)$. Then $\varphi(x, t) \geq 0$ for all $(x, t) \in(\bar{D})$.

Proof. Let there exists $\left(x_{p}, t_{q}\right) \in \bar{D}$, for some positive integer $\mathrm{p}$ and $\mathrm{q}$ such that

$$
\varphi\left(x_{p}, t_{q}\right)=\min _{(x, t) \in \bar{D}} \varphi(x, t),
$$

and also assume that $\varphi\left(x_{p}, t_{q}\right)<0$. From our assumption it is clear that $\left(x_{p}, t_{q}\right) \notin(\bar{D}-D)$, which implies that $\left(x_{p}, t_{q}\right) \in D$. Since at the point $\left(x_{p}, t_{q}\right)$ function $\varphi$ attains minimum, so $\varphi_{t}=\varphi_{x}=0, \varphi_{x x} \geq 0$ at point $\left(x_{p}, t_{q}\right)$ and $b(x)+c(x)+d(x)>0, c(x)<0$ and $d(x)<0$ for all $x \in[0,1]$. Using differential operator $\mathcal{L}_{\varepsilon}$ on $\varphi(x, t)$, from
(3) we have

$$
\begin{aligned}
& \text { for } 0<x_{p} \leq \delta \text { and } 0<t_{q} \leq T, \\
& \qquad \begin{aligned}
\mathcal{L}_{\varepsilon} \varphi\left(x_{p}, t_{q}\right)=\frac{\partial \varphi}{\partial t}-\varepsilon \frac{\partial^{2} \varphi}{\partial x^{2}}+a\left(x_{p}\right) \frac{\partial \varphi}{\partial x}+b\left(x_{p}\right) \varphi\left(x_{p}, t_{q}\right) \\
\quad+d\left(x_{p}\right) \varphi\left(x_{p}+\eta, t_{q}\right) \\
=-\varepsilon \frac{\partial^{2} \varphi}{\partial x^{2}}+b\left(x_{p}\right) \varphi\left(x_{p}, t_{q}\right)+d\left(x_{p}\right) \varphi\left(x_{p}+\eta, t_{q}\right) \\
=-\varepsilon \frac{\partial^{2} \varphi}{\partial x^{2}}+b\left(x_{p}\right) \varphi\left(x_{p}, t_{q}\right)+d\left(x_{p}\right) \varphi\left(x_{p}+\eta, t_{q}\right) \\
\quad+d\left(x_{p}\right) \varphi\left(x_{p}, t_{q}\right)-d\left(x_{p}\right) \varphi\left(x_{p}, t_{q}\right) \\
=-\varepsilon \frac{\partial^{2} \varphi}{\partial x^{2}}+\left(b\left(x_{p}\right)+d\left(x_{p}\right)\right) \varphi\left(x_{p}, t_{q}\right) \\
\quad+d\left(x_{p}\right)\left(\varphi\left(x_{p}+\eta, t_{q}\right)-\varphi\left(x_{p}, t_{q}\right)\right) \\
<0,
\end{aligned}
\end{aligned}
$$

for $\delta<x_{p}<1-\eta$ and $0<t_{q} \leq T$

$$
\begin{aligned}
\mathcal{L}_{\varepsilon} \varphi\left(x_{p}, t_{q}\right)=\frac{\partial \varphi}{\partial t}-\varepsilon \frac{\partial^{2} \varphi}{\partial x^{2}}+a\left(x_{p}\right) \frac{\partial \varphi}{\partial x}+b\left(x_{p}\right) \varphi\left(x_{p}, t_{q}\right) \\
\quad+c\left(x_{p}\right) \varphi\left(x_{p}-\delta, t_{q}\right)+d\left(x_{p}\right) \varphi\left(x_{p}+\eta, t_{q}\right) \\
=-\varepsilon \frac{\partial^{2} \varphi}{\partial x^{2}}+b\left(x_{p}\right) \varphi\left(x_{p}, t_{q}\right)+c\left(x_{p}\right) \varphi\left(x_{p}-\delta, t_{q}\right) \\
\quad+d\left(x_{p}\right) \varphi\left(x_{p}+\eta, t_{q}\right) \\
=-\varepsilon \frac{\partial^{2} \varphi}{\partial x^{2}}+\left(b\left(x_{p}\right)+c\left(x_{p}\right)+d\left(x_{p}\right)\right) \varphi\left(x_{p}, t_{q}\right) \\
\quad+c\left(x_{p}\right) \varphi\left(\left(x_{p}-\delta, t_{q}\right)-\varphi\left(x_{p}, t_{q}\right)\right)+d\left(x_{p}\right) \varphi\left(\left(x_{p}+\eta, t_{q}\right)\right. \\
\left.\quad-\varphi\left(x_{p}, t_{q}\right)\right)
\end{aligned}
$$

$<0$,

for $1-\eta \leq x_{p} \leq 1$ and $0<t_{q} \leq T$

$$
\begin{aligned}
\mathcal{L}_{\varepsilon} & \varphi\left(x_{p}, t_{q}\right)=\frac{\partial \varphi}{\partial t}-\varepsilon \frac{\partial^{2} \varphi}{\partial x^{2}}+a\left(x_{p}\right) \frac{\partial \varphi}{\partial x}+b\left(x_{p}\right) \varphi\left(x_{p}, t_{q}\right) \\
& +c\left(x_{p}\right) \varphi\left(x_{p}-\delta, t_{q}\right) \\
= & -\varepsilon \frac{\partial^{2} \varphi}{\partial x^{2}}+b\left(x_{p}\right) \varphi\left(x_{p}, t_{q}\right)+c\left(x_{p}\right) \varphi\left(x_{p}-\delta, t_{q}\right) \\
= & -\varepsilon \frac{\partial^{2} \varphi}{\partial x^{2}}+b\left(x_{p}\right) \varphi\left(x_{p}, t_{q}\right)+c\left(x_{p}\right) \varphi\left(x_{p}-\delta, t_{q}\right) \\
& +c\left(x_{p}\right) \varphi\left(x_{p}, t_{q}\right)-c\left(x_{p}\right) \varphi\left(x_{p}, t_{q}\right) \\
= & -\varepsilon \frac{\partial^{2} \varphi}{\partial x^{2}}+\left(b\left(x_{p}\right)+c\left(x_{p}\right)\right) \varphi\left(x_{p}, t_{q}\right)+c\left(x_{p}\right)\left(\varphi\left(x_{p}+\eta, t_{q}\right)\right. \\
& \left.-\varphi\left(x_{p}, t_{q}\right)\right) \\
<0 &
\end{aligned}
$$

which contradict our assumption as $\mathcal{L}_{\varepsilon} \varphi(x, t) \geq 0$ in D. So we have $\varphi(x, t) \geq 0$ for all $(x, t) \in(\bar{D})$.

Lemma 2.2. If $u(x, t)$ satisfies the maximum principle then for $n=0,1$ and 2 , the derivatives of the exact solution $u(x, t)$ of 
the model problem (1) satisfy the following bound

$$
\left|\frac{\partial^{n} u(x, t)}{\partial t^{n}}\right| \leq C, \quad(x, t) \in \bar{D}
$$

where $C$ is a generic positive constant independent of $\varepsilon$.

Proof. First we prove the bound for $\mathrm{n}=0$. We have,

$$
\begin{gathered}
|u(x, t)-u(x, 0)|=\left|u(x, t)-\phi_{b}(x)\right| \leq C t, \quad \forall(x, t) \in \bar{D} \\
|u(x, t)| \leq\left|\phi_{b}(x)\right|+C t,
\end{gathered}
$$

since $\phi_{b}(x) \in C^{2}(\bar{D})$ and $\mathrm{t}$ is bounded.

$$
|u(x, t)| \leq C_{1}, \quad \text { for some constant } C_{1} .
$$

Now we have to prove the bound for $\mathrm{n}=1$,

we have $u \equiv 0$ along $x=0$ and $x=1$, therefore $u_{x} \equiv 0$ and $u_{x x} \equiv 0$. Now for $\mathrm{t}=0$,

$$
\begin{aligned}
& \frac{\partial u}{\partial t}-\varepsilon \frac{\partial^{2} u}{\partial x^{2}}+a(x) \frac{\partial u}{\partial x}+b(x) u(x, 0)+c(x) u(x-\delta, 0) \\
& +d(x) u(x+\eta, 0)=f(x, 0),
\end{aligned}
$$

using above conditions on $\mathrm{u}$, we have

$$
\begin{gathered}
\frac{\partial u(x, 0)}{\partial t}=f(x, 0) \\
\left|\frac{\partial u(x, 0)}{\partial t}\right| \leq C, \quad \text { for some constant } C .
\end{gathered}
$$

Apply the differential operator $\mathcal{L}_{\varepsilon}$ on $u_{t}(x, t)$

$$
\mathcal{L}_{\varepsilon} u_{t}(x, t)=F_{t}(x, t)
$$

which implies that,

$$
\left|\mathcal{L}_{\varepsilon} u_{t}(x, t)\right|=\left|F_{t}(x, t)\right| \leq C, \quad \forall(x, t) \in \bar{D},
$$

using maximum principle,

$$
\left|\frac{\partial u(x, t)}{\partial t}\right| \leq C, \quad \forall(x, t) \in \bar{D} .
$$

Similarly one can prove the result for $\mathrm{n}=2$.

Theorem 2.3. For $n=0,1,2,3$ and 4, the derivatives of the exact solution $u(x, t)$ of the model problem (1) satisfy the following bound

$$
\left|\frac{\partial^{n} u(x, t)}{\partial x^{n}}\right| \leq C\left(1+\varepsilon^{-n} \exp (-\beta(1-x) / \varepsilon)\right), \quad(x, t) \in \bar{D}
$$

where $C$ is a generic positive constant independent of $\varepsilon$.

Proof. The proof of this theorem can be found in [3].

\section{Numerical Scheme}

In this section, we present a numerical scheme which works nicely when the delay and advance parameters are larger than perturbation parameter. To describe the method, we consider the linear singularly perturbed partial differential equation (1) subject to the initial and interval boundary conditions (2). Let the time interval $[0, T]$ be partitioned into $N$ equal parts with constant step size $\Delta t$. Let $0=t_{0}, t_{1}, \ldots t_{N}=T$ be the mesh points. Then we have $t_{j}=j_{N}^{T}, j=0,1, \ldots, N$.

Applying backward Euler formula for time derivative in equation (1) we obtain

$$
\begin{aligned}
& \frac{U^{j}(x)-U^{j-1}(x)}{\Delta t}-\varepsilon \frac{d^{2} U^{j}(x)}{d x^{2}}+a(x) \frac{d U^{j}(x)}{d x}+b(x) U^{j}(x) \\
& +c(x) U^{j}(x-\delta)+d(x) U^{j}(x+\eta)=f\left(x, t_{j}\right),
\end{aligned}
$$

where $U^{j}=U\left(x, t_{j}\right) \simeq u\left(x, t_{j}\right)$, for $j=1,2, \ldots, N$.

After rearranging the terms in above equation, we get

$$
\begin{aligned}
& \frac{U^{j}(x)}{\Delta t}-\varepsilon \frac{d^{2} U^{j}(x)}{d x^{2}}+a(x) \frac{d U^{j}(x)}{d x}+b(x) U^{j}(x)+c(x) U^{j}(x-\delta) \\
& +d(x) U^{j}(x+\eta)=f\left(x, t_{j}\right)+\frac{U^{j-1}(x)}{\Delta t} .
\end{aligned}
$$

This can be rewritten as

$$
\mathcal{L}_{\varepsilon}^{\star} U\left(x, t_{j}\right)=G\left(x, t_{j}\right)
$$

where

$$
\mathcal{L}_{\varepsilon}^{*} U\left(x, t_{j}\right) \equiv\left\{\begin{array}{l}
-\varepsilon \frac{d^{2} U^{j}(x)}{d x^{2}}+a(x) \frac{d U^{j}(x)}{d x}+\left(b(x)+\frac{1}{\Delta t}\right) U^{j}(x) \\
+d(x) U^{j}(x+\eta), \\
\quad \text { if } 0<x \leq \delta, \\
-\varepsilon \frac{d^{2} U^{j}(x)}{d x^{2}}+a(x) \frac{d U^{j}(x)}{d x}+\left(b(x)+\frac{1}{\Delta t}\right) U^{j}(x) \\
+c(x) U^{j}(x-\delta)+d(x) U^{j}(x+\eta), \\
\quad \text { if } \delta<x<1-\eta, \\
-\varepsilon \frac{d^{2} U^{j}(x)}{d x^{2}}+a(x) \frac{d U^{j}(x)}{d x}+\left(b(x)+\frac{1}{\Delta t}\right) U^{j}(x) \\
+c(x) U^{j}(x-\delta), \\
\quad \text { if } 1-\eta \leq x \leq 1 .
\end{array}\right.
$$

and

$$
G\left(x, t_{j}\right)=\left\{\begin{array}{c}
f\left(x, t_{j}\right)+\frac{U^{j-1}(x)}{\Delta t}-c(x) \phi_{l}\left(x-\delta, t_{j}\right), \\
\text { if } 0<x \leq \delta, \\
f\left(x, t_{j}\right)+\frac{U^{j-1}(x)}{\Delta t}, \\
\text { if } \delta<x<1-\eta, \\
f\left(x, t_{j}\right)+\frac{U^{j-1}(x)}{\Delta t}-d(x) \phi_{r}\left(x+\eta, t_{j}\right), \\
\quad \text { if } 1-\eta \leq x \leq 1 .
\end{array}\right.
$$




\subsection{Finite difference operators for a non-uniform mesh}

The first order forward, backward and central difference operators in space on non-uniform mesh(adaptive mesh) points $x_{i}$ are defined as

$$
\begin{gathered}
D_{x}^{+} u_{i}=\frac{u_{i+1}-u_{i}}{x_{i+1}-x_{i}}, \quad D_{x}^{-} u_{i}=\frac{u_{i}-u_{i-1}}{x_{i}-x_{i-1}}, \\
\quad \text { and } D_{x}^{0} u_{i}=\frac{u_{i+1}-u_{i-1}}{x_{i+1}-x_{i-1}},
\end{gathered}
$$

respectively and the second order central difference operator $D_{x}^{+} D_{x}^{-}$in space is defined as

$$
D_{\chi}^{+} D_{\chi}^{-} u_{i}=\frac{2\left(D^{+} u_{i}-D^{-} u_{i}\right)}{x_{i+1}-x_{i-1}} .
$$

Using above finite difference operators on a non-uniform mesh, we have

$$
\mathcal{L}_{\varepsilon}^{*} U\left(x, t_{j}\right) \equiv\left\{\begin{array}{l}
-\varepsilon D_{x}^{+} D_{x}^{-} U^{j}(x)+a(x) D_{x}^{0} U^{j}(x)+\left(b(x)+\frac{1}{\Delta t}\right) \\
U^{j}(x)+d(x) U^{j}(x+\eta), \\
\quad \text { if } 0<x \leq \delta, \\
-\varepsilon D_{x}^{+} D_{x}^{-} U^{j}(x)+a(x) D_{x}^{0} U^{j}(x)+\left(b(x)+\frac{1}{\Delta t}\right) \\
U^{j}(x)+c(x) U^{j}(x-\delta)+d(x) U^{j}(x+\eta), \\
\quad \text { if } \delta<x<1-\eta, \\
-\varepsilon D_{x}^{+} D_{x}^{-} U^{j}(x)+a(x) D_{x}^{0} U^{j}(x)+\left(b(x)+\frac{1}{\Delta t}\right) \\
U^{j}(x)+c(x) U^{j}(x-\delta), \\
\quad \text { if } 1-\eta \leq x \leq 1 .
\end{array}\right.
$$

The initial and interval boundary conditions can be rewritten as

$$
\begin{gathered}
U(x, 0)=\phi_{b}(x), \quad x \in[0,1], \\
U\left(x, t_{j}\right)=\phi_{l}\left(x, t_{j}\right), \quad-\delta \leq x \leq 1, j=0,1, \ldots, N, \\
U\left(x, t_{j}\right)=\phi_{r}\left(x, t_{j}\right), \quad 1 \leq x \leq 1+\eta, j=0,1, \ldots, N .
\end{gathered}
$$

We solve (11) along with the conditions (12) using central difference scheme with minimum number of mesh points on uniform mesh in space direction. The presence of the singular perturbation parameter $\varepsilon$ leads to occurrences of wild oscillation in the numerical solution. In order to avoid such oscillations, a large number of mesh points are required in layer region, when $\varepsilon$ is very small. To overcome this, we generated a variable mesh using entropy function. The strategy for generating an adaptive mesh is given in the following subsection.

\subsection{Mesh Selection Strategy}

Now, we define, the entropy production equation by multiplying with an appropriate test function. From the theory of scalar conservation law, we know that $U^{2}$ is always an appropriate entropy variable and therefore $2 U\left(x, t_{j}\right)$ is a suitable multiplying test function [10]. On multiplying with the test function, we obtain

$$
\mathcal{L}_{\varepsilon}^{\star} U\left(x, t_{j}\right) \star 2 U\left(x, t_{j}\right)=G\left(x, t_{j}\right) \star 2 U\left(x, t_{j}\right) .
$$

After simplifying, equation (13) can be written as

$$
\left\{\begin{array}{l}
-\varepsilon\left(Z^{\prime \prime}-2\left(U^{\prime 2}\right)\right)+a(x) Z^{\prime}+2\left(b(x)+\frac{1}{\Delta t}\right) Z+2 U^{j} \\
\left(c(x) \phi_{l}(x-\delta)+d(x) U^{j}(x+\eta)-f\left(x, t_{j}\right)-\frac{U^{j-1}(x)}{\Delta t}\right)=0, \\
\quad \text { if } 0<x \leq \delta, \\
-\varepsilon\left(Z^{\prime \prime}-2\left(U^{\prime 2}\right)\right)+a(x) Z^{\prime}+2\left(b(x)+\frac{1}{\Delta t}\right) Z+2 U^{j} \\
\left(c(x) U^{j}(x-\delta)+d(x) U^{j}(x+\eta)-f\left(x, t_{j}\right)-\frac{U^{j-1}(x)}{\Delta t}\right)=0, \\
\quad \text { if } \delta<x<1-\eta, \\
-\varepsilon\left(Z^{\prime \prime}-2\left(U^{\prime 2}\right)\right)+a(x) Z^{\prime}+2\left(b(x)+\frac{1}{\Delta t}\right) Z+2 U^{j} \\
\left(c(x) U^{j}(x-\delta)+d(x) \phi_{r}(x+\eta)-f\left(x, t_{j}\right)-\frac{U^{j-1}(x)}{\Delta t}\right)=0, \\
\quad \text { if } 1-\eta \leq x \leq 1,
\end{array}\right.
$$

where $Z=U^{2}$.

The above equation can be rewritten as

$$
\left\{\begin{array}{l}
-\varepsilon Z^{\prime \prime}+a(x) Z^{\prime}+2 U^{j}\left(c(x) \phi_{l}(x-\delta)+d(x) U^{j}(x+\eta)\right. \\
\left.-f\left(x, t_{j}\right)-\frac{U^{j-1}(x)}{\Delta t}\right)=-2\left(b(x)+\frac{1}{\Delta t}\right) Z-2 \varepsilon\left(U^{\prime 2}\right), \\
\text { if } 0<x \leq \delta, \\
-\varepsilon Z^{\prime \prime}+a(x) Z^{\prime}+2 U^{j}\left(c(x) U^{j}(x-\delta)+d(x) U^{j}(x+\eta)\right. \\
\left.-f\left(x, t_{j}\right)-\frac{U^{j-1}(x)}{\Delta t}\right)=-2\left(b(x)+\frac{1}{\Delta t}\right) Z-2 \varepsilon\left(U^{\prime 2}\right), \\
\text { if } \delta<x<1-\eta, \\
-\varepsilon Z^{\prime \prime}+a(x) Z^{\prime}+2 U^{j}\left(c(x) U^{j}(x-\delta)+d(x) \phi_{r}(x+\eta)\right. \\
\left.-f\left(x, t_{j}\right)-\frac{U^{j-1}(x)}{\Delta t}\right)=-2\left(b(x)+\frac{1}{\Delta t}\right) Z-2 \varepsilon\left(U^{\prime 2}\right) . \\
\text { if } 1-\eta \leq x \leq 1,
\end{array}\right.
$$

The right-hand side of the equation(14) is considered as our entropy function and is always negative for all values $x \in[0,1]$. As we know that, if we solve equation (11) by using central difference method, we get oscillations inside and near the boundary layer region. We calculate the discrete analogue of left hand side part in (14) using the same central difference operator by taking $Z_{i}=U_{i}^{2}$, where $U_{i}$ is 
the central difference computed solution of equation (11). If we write the right hand side part of (14) at the mesh point $\left(x_{i}, t_{j}\right)$, as

$$
\begin{aligned}
& -2\left(b\left(x_{i}\right)+\frac{1}{\Delta t}\right)\left(U_{i+1, j} \cdot U_{i-1, j}\right)-2 \varepsilon\left(\frac{U_{i, j}-U_{i-1, j}}{x_{i}-x_{i-1}}\right) \\
& \left(\frac{U_{i+1, j}-U_{i, j}}{x_{i+1}-x_{i}}\right),
\end{aligned}
$$

we get the positive value whenever the oscillations occur, where $U_{i}$ is the central difference computed solution. To generate the variable mesh, first we calculate entropy function with minimum number of initial uniform mesh points in space direction. To handle the delay and advance terms, we construct a special mesh, so that the terms containing the shifts lie on nodal point after discretization on uniform mesh. We find out the mesh point, where the entropy is maximum and positive. We add mesh points, one to the left and other to the right side of the mesh point where entropy is maximum and positive. Now, we compute the solution with newly generated mesh points (non uniform mesh) using central difference method and check whether the entropy is positive or negative through out the interval of integration. If the entropy is positive, we pick up the mesh point where the entropy is maximum and positive and we repeat the process of adding mesh points both sides. We repeat this process till we get entropy negative through out the interval of integration.

\section{Stability and Error analysis}

Use the difference operators for a non-uniform mesh in (11), since $h_{i}=x_{i+1}-x_{i}, h_{i-1}=x_{i}-x_{i-1}$ and $h_{i}+h_{i-1}=$ $x_{i+1}-x_{i-1}$. For $1<k, l<M$, we have

$$
\left\{\begin{array}{l}
-\varepsilon \frac{2\left(D^{+} U_{i, j}-D^{-} U_{i, j}\right)}{x_{i+1}-x_{i-1}}+a_{i} \frac{U_{i+1, j}-U_{i-1, j}}{x_{i+1}-x_{i-1}} \\
+\left(b_{i}+\frac{1}{\Delta t}\right) U_{i, j}+d_{i} U_{i+l, j}, \\
\quad \text { if } i=1,2, \ldots, k, \\
-\varepsilon \frac{2\left(D^{+} U_{i, j}-D^{-} U_{i, j}\right)}{x_{i+1}-x_{i-1}}+a_{i} \frac{U_{i+1, j}-U_{i-1, j}}{x_{i+1}-x_{i-1}} \\
+\left(b_{i}+\frac{1}{\Delta t}\right) U_{i, j}+c_{i} U_{i-k, j}+d_{i} U_{i+l, j}, \\
\quad \text { if } i=k+1, k+2, \ldots, M-l-1, \\
-\varepsilon \frac{2\left(D^{+} U_{i, j}-D^{-} U_{i, j}\right)}{x_{i+1}-x_{i-1}}+a_{i} \frac{U_{i+1, j}-U_{i-1, j}}{x_{i+1}-x_{i-1}} \\
+\left(b_{i}+\frac{1}{\Delta t}\right) U_{i, j}+d_{i} U_{i+l, j}, \\
\quad \text { if } i=M-l, M-l+1, \ldots, M,
\end{array}\right.
$$

and

$$
G_{i, j}=\left\{\begin{array}{c}
f_{i, j}+\frac{U_{i, j-1}}{\Delta t}-c_{i} \phi_{l}(i-k, j), \\
\quad \text { if } i=1,2, \ldots, k, \\
f_{i, j}+\frac{U_{i, j-1}}{\Delta t}, \\
\quad \text { if } i=k+1, k+2, \ldots, M-l-1, \\
f_{i, j}+\frac{U_{i, j-1}}{\Delta t}-d_{i} \phi_{r}(i+l, j), \\
\quad \text { if } i=M-l, M-l+1, \ldots, M .
\end{array}\right.
$$

On rearrangement, the Equation (16)-(17) can be written as

$E_{i} U_{i-1, j}+F_{i} U_{i, j}+H_{i} U_{i+1, j}+I_{i} U_{i-k, j}+J_{i} U_{i+l, j}=f_{i, j}+\frac{U_{i, j-1}}{\Delta t}$,

where

$$
\begin{aligned}
E_{i} & =\left|\frac{-2 \varepsilon}{h_{i-1}\left(h_{i}+h_{i-1}\right)}-\frac{a_{i}}{h_{i}+h_{i-1}}\right| \\
F_{i} & =\left|\frac{2 \varepsilon}{h_{i}\left(h_{i}+h_{i-1}\right)}+\frac{2 \varepsilon}{h_{i-1}\left(h_{i}+h_{i-1}\right)}+b_{i}+\frac{1}{\Delta t}\right| \\
H_{i} & =\left|\frac{-2 \varepsilon}{h_{i}\left(h_{i}+h_{i-1}\right)}+\frac{a_{i}}{h_{i}+h_{i-1}}\right| \\
I_{i} & =\left|c_{i}\right| \\
J_{i} & =\left|d_{i}\right|
\end{aligned}
$$

and

$$
\left|E_{i}\right|+\left|H_{i}\right|=\frac{2 \varepsilon}{h_{i} h_{i-1}},
$$

provided $\left|a_{i}\right| \leq 2 \varepsilon / h_{i-1}$ and $\left|a_{i}\right| \leq 2 \varepsilon / h_{i}$.

For $i=1,2, \ldots, k$ and $j=1,2, \ldots, N$,

$$
\begin{gathered}
E_{i} U_{i-1, j}+F_{i} U_{i, j}+H_{i} U_{i+1, j}+J_{i} U_{i+l, j}=f_{i, j} \\
+\frac{U_{i, j-1}}{\Delta t}-c_{i} \phi_{l}(i-k, j) \\
\left|F_{i}\right|-\left|E_{i}\right|-\left|H_{i}\right|-\left|J_{i}\right|=\left|\frac{2 \varepsilon}{h_{i} h_{i-1}}+b_{i}+\frac{1}{\Delta t}\right|-\left|\frac{2 \varepsilon}{h_{i} h_{i-1}}\right|-d_{i} \\
=b_{i}+\frac{1}{\Delta t}-d_{i}
\end{gathered}
$$

since $b_{i}>0$ and $d_{i}<0$ on $[0,1]$ and $\Delta t$ is step size in temporal direction. We have

$$
\left|F_{i}\right|>\left|E_{i}\right|+\left|H_{i}\right|+\left|J_{i}\right| \cdot
$$

For $i=k+1, k+2, \ldots, M-l-1$, and $j=1,2, \ldots, N$,

$$
\begin{aligned}
E_{i} U_{i-1, j}+F_{i} U_{i, j}+H_{i} U_{i+1, j}+I_{i} U_{i-k, j} & +J_{i} U_{i+l, j}=f_{i, j}+\frac{U_{i, j-1}}{\Delta t} \\
\left|F_{i}\right|-\left|E_{i}\right|-\left|H_{i}\right|-\left|I_{i}\right|-\left|J_{i}\right| & =\left|\frac{2 \varepsilon}{h_{i} h_{i-1}}+b_{i}+\frac{1}{\Delta t}\right| \\
& -\left|\frac{2 \varepsilon}{h_{i} h_{i-1}}\right|-c_{i}-d_{i} \\
& =b_{i}+\frac{1}{\Delta t}-c_{i}-d_{i},
\end{aligned}
$$


since $b_{i}>0, c_{i}<0$ and $d_{i}<0$ on $[0,1]$ and $\Delta t$ is step size in temporal direction. We have

$$
\left|F_{i}\right|>\left|E_{i}\right|+\left|H_{i}\right|+\left|I_{i}\right|+\left|J_{i}\right| .
$$

For $i=M-l, M-l+1, \ldots, M$, and $j=1,2, \ldots, N$,

$$
\begin{aligned}
& E_{i} U_{i-1, j}+F_{i} U_{i, j}+H_{i} U_{i+1, j}+I_{i} U_{i+l, j}=f_{i, j} \\
& +\frac{U_{i, j-1}}{\Delta t}-d_{i} \phi_{r}(i+l, j)
\end{aligned}
$$

$$
\begin{aligned}
\left|F_{i}\right|-\left|E_{i}\right|-\left|H_{i}\right|-\left|I_{i}\right| & =\left|\frac{2 \varepsilon}{h_{i} h_{i-1}}+b_{i}+\frac{1}{\Delta t}\right|-\left|\frac{2 \varepsilon}{h_{i} h_{i-1}}\right|-c_{i} \\
& =b_{i}+\frac{1}{\Delta t}-c_{i}
\end{aligned}
$$

since $b_{i}>0$ and $c_{i}<0$ on $[0,1]$ and $\Delta t$ is step size in temporal direction. We have

$$
\left|F_{i}\right|>\left|E_{i}\right|+\left|H_{i}\right|+\left|I_{i}\right|,
$$

which shows that the given system is diagonally dominant. The scheme is stable and the unknown $U_{i . j}$ must be solved at each time level.

Truncation error : Let $M^{\star}$ is number of mesh interval on an adaptive mesh obtained using entropy function concept. If $x_{i}$ is $i^{\text {th }}$ mesh point on $M^{\star}$. We define left hand and right hand side distance by

$$
h_{i-1}=x_{i}-x_{i-1}, \quad h_{i}=x_{i+1}-x_{i} .
$$

Using Taylors series expansion

$$
\begin{aligned}
& u\left(x_{i}-h_{i-1}, t_{j}\right)=u\left(x_{i}, t_{j}\right)-h_{i-1} \frac{\partial u\left(x_{i}, t_{j}\right)}{\partial x}+\frac{h_{i-1}^{2}}{2 !} \frac{\partial^{2} u\left(x_{i}, t_{j}\right)}{\partial x^{2}} \\
& -\frac{h_{i-1}^{3}}{3 !} \frac{\partial^{3} u\left(x_{i}, t_{j}\right)}{\partial x^{3}}+\ldots \\
& u\left(x_{i}+h_{i}, t_{j}\right)=u\left(x_{i}, t_{j}\right)+h_{i} \frac{\partial u\left(x_{i}, t_{j}\right)}{\partial x}+\frac{h_{i}^{2}}{2 !} \frac{\partial^{2} u\left(x_{i}, t_{j}\right)}{\partial x^{2}} \\
& +\frac{h_{i}^{3}}{3 !} \frac{\partial^{3} u\left(x_{i}, t_{j}\right)}{\partial x^{3}}+\ldots
\end{aligned}
$$

and

$$
\begin{aligned}
& u\left(x_{i}, t_{j}-\Delta t\right)=u\left(x_{i}, t_{j}\right)-\Delta t \frac{\partial u\left(x_{i}, t_{j}\right)}{\partial t}+\frac{\Delta t^{2}}{2 !} \frac{\partial^{2} u\left(x_{i}, t_{j}\right)}{\partial t^{2}} \\
& -\frac{\Delta t^{3}}{3 !} \frac{\partial^{3} u\left(x_{i}, t_{j}\right)}{\partial t^{3}}+\ldots,
\end{aligned}
$$

using these expansions in Eq. (18), we get

$$
\begin{aligned}
& \left(\frac{-2 \varepsilon}{h_{i-1}\left(h_{i}+h_{i-1}\right)}-\frac{a_{i}}{h_{i}+h_{i-1}}\right)\left(u\left(x_{i}, t_{j}\right)-h_{i-1} \frac{\partial u\left(x_{i}, t_{j}\right)}{\partial x}\right. \\
& \left.+h_{i-1}^{2} \frac{\partial^{2} u\left(x_{i}, t_{j}\right)}{\partial x^{2}}-h_{i-1}^{3} \frac{\partial^{3} u\left(x_{i}, t_{j}\right)}{\partial x^{3}}+\ldots\right)+\left(\frac{2 \varepsilon}{h_{i}\left(h_{i}+h_{i-1}\right)}\right. \\
& \left.+\frac{2 \varepsilon}{h_{i-1}\left(h_{i}+h_{i-1}\right)}+b_{i}+\frac{1}{\Delta t}\right) u\left(x_{i}, t_{j}\right)+\left(\frac{-2 \varepsilon}{h_{i}\left(h_{i}+h_{i-1}\right)}\right. \\
& \left.+\frac{a_{i}}{h_{i}+h_{i-1}}\right)\left(u\left(x_{i}, t_{j}\right)+h_{i} \frac{\partial u\left(x_{i}, t_{j}\right)}{\partial x}+h_{i}^{2} \frac{\partial^{2} u\left(x_{i}, t_{j}\right)}{\partial x^{2}}\right. \\
& \left.+h_{i}^{3} \frac{\partial^{3} u\left(x_{i}, t_{j}\right)}{\partial x^{3}}+\ldots\right)+c_{i} u\left(x_{i}-\delta, t_{j}\right)+d_{i} u\left(x_{i}+\eta, t_{j}\right) \\
& -\frac{1}{\Delta t}\left(u\left(x_{i}, t_{j}\right)-\Delta t \frac{\partial u\left(x_{i}, t_{j}\right)}{\partial t}+\Delta t^{2} \frac{\partial^{2} u\left(x_{i}, t_{j}\right)}{\partial t^{2}}\right. \\
& \left.-\Delta t^{3} \frac{\partial^{3} u\left(x_{i}, t_{j}\right)}{\partial t^{3}}+\ldots\right)=0 .
\end{aligned}
$$

After simplification the terms, the truncation error is

$$
\begin{aligned}
& \text { T.E. }=\frac{\partial u\left(x_{i}, t_{j}\right)}{\partial t}-\varepsilon \frac{\partial^{2} u\left(x_{i}, t_{j}\right)}{\partial x^{2}}+b\left(x_{i}\right) u\left(x_{i}, t_{j}\right) \\
& +a\left(x_{i}\right) \frac{\partial u\left(x_{i}, t_{j}\right)}{\partial x}+c\left(x_{i}\right) u\left(x_{i}-\delta, t_{j}\right)+d\left(x_{i}\right) u\left(x_{i}+\eta, t_{j}\right) \\
& -f\left(x_{i}, t_{j}\right)+\frac{a\left(x_{i}\right)\left(h_{i}-h_{i-1}\right)}{2} \frac{\partial^{2} u\left(x_{i}, t_{j}\right)}{\partial x^{2}}-\frac{\Delta t}{2} \frac{\partial^{2} u\left(x_{i}, t_{j}\right)}{\partial t^{2}} \\
& +\left(\frac{-\varepsilon\left(h_{i}-h_{i-1}\right)}{3}+\frac{a\left(x_{i}\right)\left(h_{i-1}^{3}+h_{i}^{3}\right)}{h_{i-1}+h_{i}}\right) \frac{\partial^{3} u\left(x_{i}, t_{j}\right)}{\partial x^{3}} \\
& +\frac{\Delta t^{2}}{6} \frac{\partial^{3} u\left(x_{i}, t_{j}\right)}{\partial t^{3}},
\end{aligned}
$$

which can further be simplified to

$$
\begin{aligned}
& \text { T.E. }=\frac{a\left(x_{i}\right)\left(h_{i}-h_{i-1}\right)}{2} \frac{\partial^{2} u\left(x_{i}, t_{j}\right)}{\partial x^{2}}-\frac{\Delta t}{2} \frac{\partial^{2} u\left(x_{i}, t_{j}\right)}{\partial t^{2}} \\
& +\left(\frac{-\varepsilon\left(h_{i}-h_{i-1}\right)}{3}+\frac{a\left(x_{i}\right)\left(h_{i-1}^{3}+h_{i}^{3}\right)}{h_{i-1}+h_{i}}\right) \frac{\partial^{3} u\left(x_{i}, t_{j}\right)}{\partial x^{3}} \\
& +\frac{\Delta t^{2}}{6} \frac{\partial^{3} u\left(x_{i}, t_{j}\right)}{\partial t^{3}},
\end{aligned}
$$

as $h_{i-1}, h_{i} \rightarrow 0$ and $\Delta t \rightarrow 0$ the T.E. $\rightarrow 0$, which shows that scheme is consistent and the truncation error of the scheme is of order $O\left(h_{i}-h_{i-1}, \Delta t\right)$. The Lax equivalence theorem says that a finite difference approximation for a properly posed partial differential equation satisfying consistency and stability are necessary and sufficient conditions for convergence.

\section{Numerical results}

To demonstrate the efficiency and applicability of the proposed numerical scheme, numerical experiments are carried out for three test problems of singularly perturbed partial differential equations with large shifts parameters. The 
exact solution of the problems are not known, so the maximum point wise errors are calculated using the following double mesh principle [6]:

$$
E_{\varepsilon}^{M^{*}, N}=\max _{0 \leq i, j \leq M^{*}, N}\left|U^{M^{*}, N}\left(x_{i}, t_{j}\right)-U^{2 M^{*}, 2 N}\left(x_{2 i}, t_{j}\right)\right|,
$$

where $U^{M^{*}, N}\left(x_{i}, t_{j}\right)$, denote the numerical solution obtained on a mesh containing $M^{\star}+1$ points in spatial direction and $N+1$ points in temporal direction.

The numerical rate of convergence is calculated using the formula

$$
R_{\varepsilon}^{M^{*}, N}=\frac{\log \left|E_{\varepsilon}^{M^{*}, N}-E_{\varepsilon}^{2 M^{*}, 2 N}\right|}{\log 2} .
$$

We use the notation $M, N$ for mesh intervals in space and time direction, on uniform mesh and $M^{\star}$ for final adaptive mesh intervals in space direction.

Example 5.1[3] Consider the following singularly perturbed partial differential equation :

$$
\begin{aligned}
& \frac{\partial u}{\partial t}-\varepsilon \frac{\partial^{2} u}{\partial x^{2}}+\left(2-x^{2}\right) \frac{\partial u}{\partial x}+(x+3) u(x, t)-u(x-\delta, t) \\
& -2 u(x+\eta, t)=10 t^{2} e^{-t} x(1-x),
\end{aligned}
$$

where $(x, t) \in(0,1) \times(0,3]$ and is subject to the following interval boundary conditions and the initial condition,

$$
\begin{aligned}
& u(x, t)=0, \quad \forall(x, t) \in \Gamma_{l}=\{(x, t):-\delta \leq x \leq 0 \\
& \text { and } 0 \leq t \leq T\}, \\
& u(x, t)=0, \quad \forall(x, t) \in \Gamma_{r}=\{(x, t): 1 \leq x \leq 1+\eta \\
& \text { and } 0 \leq t \leq T\}, \\
& \qquad u(x, t)=0, \quad \forall x \in[0,1] .
\end{aligned}
$$

The adaptive mesh generation with $\varepsilon=2^{-10}$, for this example is plotted in Figure 1. The numerical solution of the central finite difference scheme on uniform mesh and on adaptive mesh for three different time levels with $\varepsilon=$ $2^{-14}, \delta=0.2$ and $\eta=0.4$, for this example, is plotted in Figure $2 \mathrm{a}$ and Figure $2 \mathrm{~b}$. To examine the effect of shift parameters on the boundary layer behaviour of the solution, surface plot of the solution of example 5.1 for different values of $\varepsilon$ and $\delta$ and $\eta$, using adaptive mesh is plotted in Figure $3 \mathrm{a}$ and Figure $3 \mathrm{~b}$. The maximum point wise errors and rate of convergence are presented in Table 1 for this boundary value problem for different values of perturbation parameter $\varepsilon$.
Table 1: Maximum point wise errors of the solution and corresponding rate of convergence for Example 5.1 by taking $\delta=0.6, \eta=0.7$, $M=10$ and $N=20$ for different values of $\varepsilon$.

\begin{tabular}{cccc}
\hline$\varepsilon$ & $\begin{array}{c}\text { Generated mesh } \\
\left(M^{\star}\right)\end{array}$ & Max. error & $\begin{array}{c}\text { Rate of Convergence } \\
\left(R^{M^{\star}, N}\right)\end{array}$ \\
\hline $2^{-10}$ & 22 & $2.3422 \mathrm{e}-02$ & 2.2166 \\
$2^{-11}$ & 24 & $2.4004 \mathrm{e}-02$ & 2.2086 \\
$2^{-12}$ & 26 & $2.4260 \mathrm{e}-02$ & 2.2037 \\
$2^{-13}$ & 28 & $2.4377 \mathrm{e}-02$ & 2.2012 \\
$2^{-14}$ & 30 & $2.4432 \mathrm{e}-02$ & 2.1910 \\
$2^{-15}$ & 32 & $2.4460 \mathrm{e}-02$ & 2.1993 \\
$2^{-16}$ & 34 & $2.4473 \mathrm{e}-02$ & 2.1991 \\
$2^{-17}$ & 36 & $2.4479 \mathrm{e}-02$ & 2.1989 \\
$2^{-18}$ & 38 & $2.4483 \mathrm{e}-02$ & 2.1989 \\
$2^{-19}$ & 40 & $2.4484 \mathrm{e}-02$ & 2.1988 \\
$2^{-20}$ & 42 & $2.4485 \mathrm{e}-02$ & 2.1988 \\
$2^{-21}$ & 44 & $2.4485 \mathrm{e}-02$ & 2.1988 \\
$2^{-22}$ & 46 & $2.4486 \mathrm{e}-02$ & 2.1988 \\
$2^{-23}$ & 48 & $2.4486 \mathrm{e}-02$ & 2.1988 \\
$2^{-24}$ & 50 & $2.4486 \mathrm{e}-02$ & 2.1988 \\
$2^{-25}$ & 52 & $2.4486 \mathrm{e}-02$ & 2.1988 \\
$2^{-26}$ & 54 & $2.4486 \mathrm{e}-02$ & 2.1988 \\
$2^{-27}$ & 56 & $2.4486 \mathrm{e}-02$ & 2.1988 \\
$2^{-28}$ & 58 & $2.4486 \mathrm{e}-02$ & 2.1988 \\
$2^{-29}$ & 60 & $2.4486 \mathrm{e}-02$ & 2.1988 \\
$2^{-30}$ & 62 & $2.4486 \mathrm{e}-02$ & 2.1988 \\
\hline
\end{tabular}

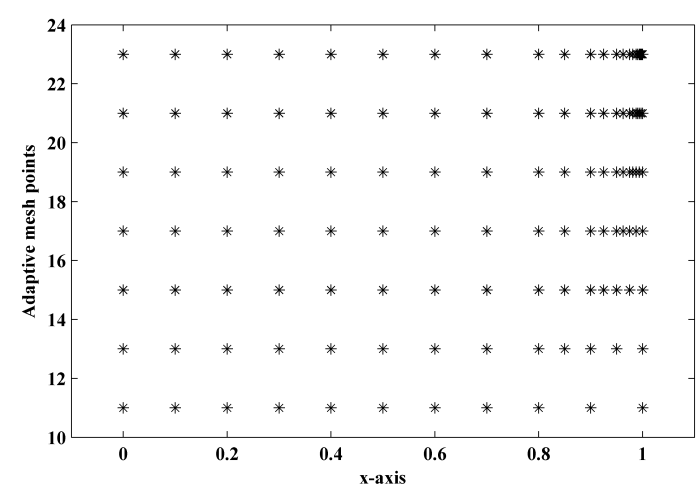

Fig. 1: Adaptive mesh generation for example 5.1 with $\varepsilon=2^{-10}$, $\delta=0.6, \eta=0.7, \mathrm{M}=10$ (initially) and $\mathrm{N}=20$.

Example 5.2[3] Consider the following singularly perturbed partial differential equation :

$$
\begin{aligned}
& \frac{\partial u}{\partial t}-\varepsilon \frac{\partial^{2} u}{\partial x^{2}}+\left(1+x+x^{2}\right) \frac{\partial u}{\partial x}+\left(1+x^{2}\right) u(x, t) \\
& -\left(\frac{1}{4}+\frac{x^{2}}{2}\right) u(x-\delta, t)-\frac{1}{4} u(x+\eta, t)=\sin \pi x(1-x),
\end{aligned}
$$

where $(x, t) \in(0,1) \times(0,1]$ and is subject to the following interval boundary conditions and the initial condition,

$$
\begin{aligned}
& u(x, t)=0, \quad \forall(x, t) \in \Gamma_{l}=\{(x, t):-\delta \leq x \leq 0 \\
& \text { and } 0 \leq t \leq T\},
\end{aligned}
$$




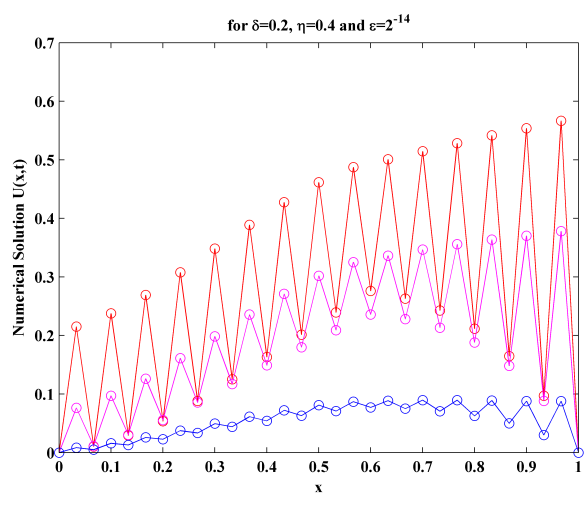

(a) Uniform mesh.

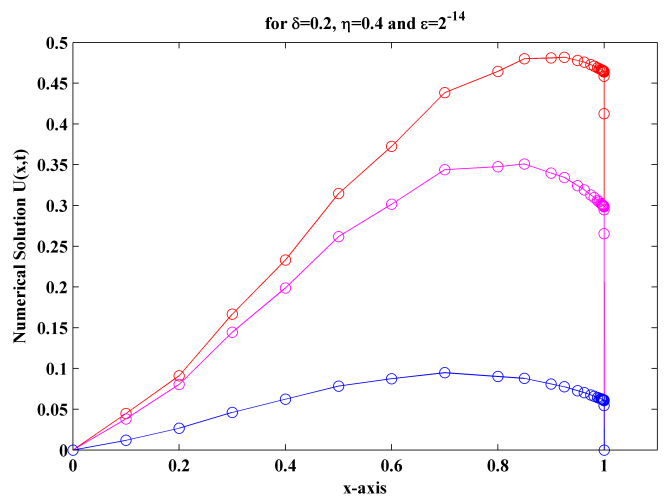

(b) Adaptive mesh.

Fig. 2: Numerical solution of example 5.1, using uniform mesh and adaptive mesh for three different time level with $\varepsilon=2^{-14}, \delta=$ $0.2, \eta=0.4, \mathrm{M}=30$ and $\mathrm{N}=20$.

$$
\begin{aligned}
& u(x, t)=0, \quad \forall(x, t) \in \Gamma_{r}=\{(x, t): 1 \leq x \leq 1+\eta \\
& \text { and } \quad 0 \leq t \leq T\},
\end{aligned}
$$

$$
u(x, t)=0, \quad \forall x \in[0,1] .
$$

The adaptive mesh generation with $\varepsilon=2^{-20}$, for this example is plotted in Figure 4. The numerical solution of the central finite difference scheme on uniform mesh and on adaptive mesh for three different time level with $\varepsilon=2^{-22}$, $\delta=0.7$ and $\eta=0$, for this example, is plotted in Figure 5a and Figure 5b. To examine the effect of shift parameters on the boundary layer behaviour of the solution, surface plot of the solution of example 5.2 for different values of $\varepsilon$ and $\delta$ and $\eta$, using adaptive mesh is plotted in Figure 6a and Figure $6 \mathrm{~b}$. The maximum point wise errors and rate of convergence are presented in Table 2, 3, 4 for this boundary value problem for different values of perturbation parameter $\varepsilon$.

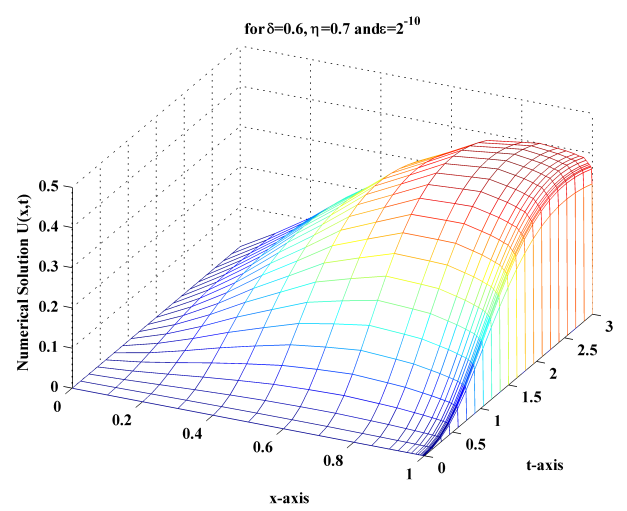

(a) $M^{\star}=22, N=20$

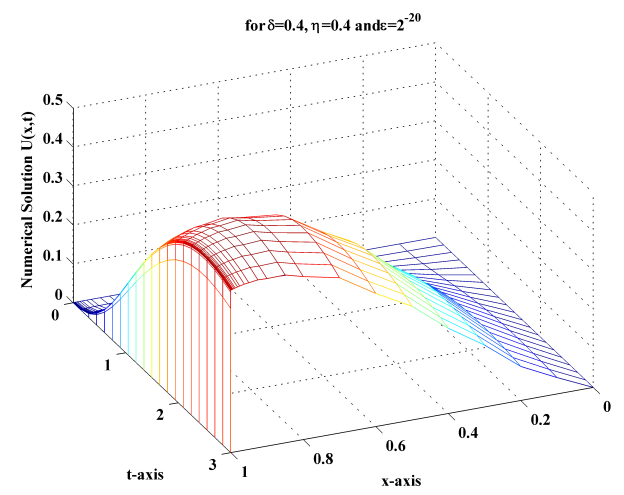

(b) $M^{\star}=42, N=20$

Fig. 3: Surface plot of example 5.1, using adaptive mesh for different values of $\epsilon, \delta$ and $\eta$.

Table 2: Maximum point wise errors of the solution and corresponding rate of convergence for Example 5.2 by taking $\delta=0.6, \eta=0.7$, $M=10$ and $N=20$ for different values of $\varepsilon$.

\begin{tabular}{cccc}
\hline$\varepsilon$ & $\begin{array}{c}\text { Generated mesh } \\
\left(M^{\star}\right)\end{array}$ & Max. error & $\begin{array}{c}\text { Rate of Convergence } \\
\left(R^{M^{\star}, N}\right)\end{array}$ \\
\hline $2^{-10}$ & 26 & $1.0006 \mathrm{e}-02$ & 1.3435 \\
$2^{-15}$ & 36 & $1.0058 \mathrm{e}-02$ & 1.3392 \\
$2^{-20}$ & 46 & $1.3391 \mathrm{e}-02$ & 1.3391 \\
$2^{-25}$ & 58 & $1.0059 \mathrm{e}-02$ & 1.3391 \\
$2^{-30}$ & 68 & $1.0059 \mathrm{e}-02$ & 1.3391 \\
\hline
\end{tabular}

Example 5.3[3] Consider the following singularly perturbed partial differential equation :

$$
\begin{aligned}
& \frac{\partial u}{\partial t}-\varepsilon \frac{\partial^{2} u}{\partial x^{2}}+\left(1-\frac{x^{2}}{2}\right) \frac{\partial u}{\partial x}+(x+6) u(x, t)-4 u(x-\delta, t) \\
& -u(x+\eta, t)=x(1-x)
\end{aligned}
$$


Table 3: Maximum point wise errors of the solution and corresponding rate of convergence for Example 5.2 by taking $\delta=0, \eta=0.5$, $M=10$ and $N=20$ for different values of $\varepsilon$.

\begin{tabular}{cccc}
\hline$\varepsilon$ & $\begin{array}{c}\text { Generated mesh } \\
\left(M^{\star}\right)\end{array}$ & Max. error & $\begin{array}{c}\text { Rate of Convergence } \\
\left(R^{M^{\star}, N}\right)\end{array}$ \\
\hline $2^{-10}$ & 26 & $1.1246 \mathrm{e}-02$ & 1.3077 \\
$2^{-15}$ & 36 & $1.1300 \mathrm{e}-02$ & 1.3033 \\
$2^{-20}$ & 46 & $1.1302 \mathrm{e}-02$ & 1.3031 \\
$2^{-25}$ & 58 & $1.1302 \mathrm{e}-02$ & 1.3031 \\
$2^{-30}$ & 68 & $1.1302 \mathrm{e}-02$ & 1.3031 \\
\hline
\end{tabular}

Table 4: Maximum point wise errors of the solution and corresponding rate of convergence for Example 5.2 by taking $\delta=0.7, \eta=0$, $M=10$ and $\mathrm{N}=20$ for different values of $\varepsilon$.

\begin{tabular}{cccc}
\hline$\varepsilon$ & $\begin{array}{c}\text { Generated mesh } \\
\left(M^{\star}\right)\end{array}$ & Max. error & $\begin{array}{c}\text { Rate of Convergence } \\
\left(R^{M^{\star}, N}\right)\end{array}$ \\
\hline $2^{-10}$ & 26 & $1.0145 \mathrm{e}-02$ & 1.3435 \\
$2^{-15}$ & 36 & $1.0193 \mathrm{e}-02$ & 1.3392 \\
$2^{-20}$ & 46 & $1.0194 \mathrm{e}-02$ & 1.3391 \\
$2^{-25}$ & 58 & $1.0194 \mathrm{e}-02$ & 1.3391 \\
$2^{-30}$ & 68 & $1.0194 \mathrm{e}-02$ & 1.3391 \\
\hline
\end{tabular}

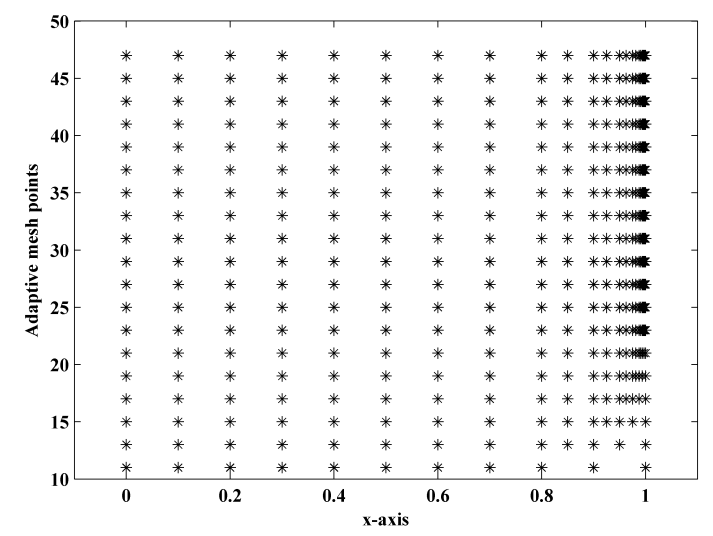

Fig. 4: Adaptive mesh generation for example 5.2 with $\varepsilon=2^{-20}$, $\delta=0, \eta=0.5, \mathrm{M}=10$ (initially) and $\mathrm{N}=20$.

where $(x, t) \in(0,1) \times(0,3]$ and is subject to the following interval boundary conditions and the initial condition,

$$
\begin{aligned}
& u(x, t)=0, \quad \forall(x, t) \in \Gamma_{l}=\{(x, t):-\delta \leq x \leq 0 \\
& \text { and } 0 \leq t \leq T\},
\end{aligned}
$$

$$
\begin{aligned}
& u(x, t)=0, \quad \forall(x, t) \in \Gamma_{r}=\{(x, t): 1 \leq x \leq 1+\eta \\
& \text { and } \quad 0 \leq t \leq T\},
\end{aligned}
$$

$$
u(x, t)=0, \quad \forall x \in[0,1] .
$$

The adaptive mesh generation with $\varepsilon=2^{-30}$, for this example is plotted in Figure 7. The numerical solution of the

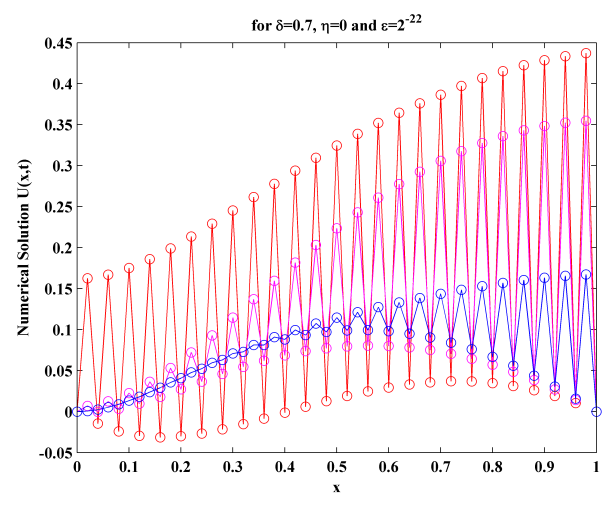

(a) Uniform mesh.

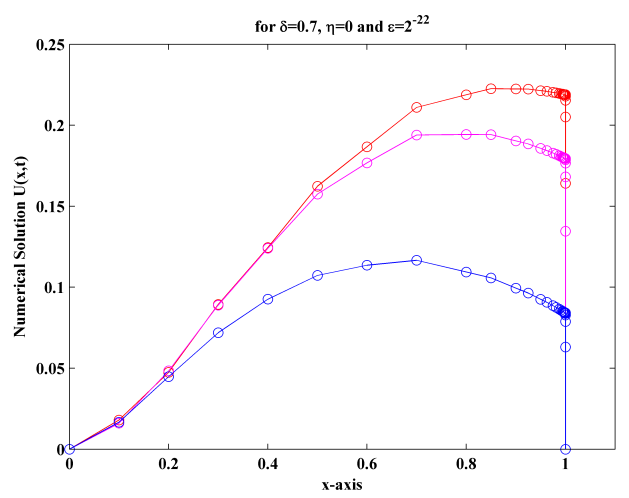

(b) Adaptive mesh.

Fig. 5: Numerical solution of example 5.2, using uniform mesh and adaptive mesh for three different time level with $\varepsilon=2^{-22}, \delta=$ $0.7, \eta=0, M=50$ and $\mathrm{N}=20$.

central finite difference scheme on uniform mesh and on adaptive mesh for three different time level with $\delta=0.1$ and $\eta=0.2$, for this example, is plotted in Figure $8 \mathrm{a}$ and Figure $8 \mathrm{~b}$. To examine the effect of shift parameters on the boundary layer behaviour of the solution, surface plot of the solution of example 5.3 for different values of $\varepsilon$ and $\delta$ and $\eta$, using adaptive mesh is plotted in Figure 9a and Figure $9 \mathrm{~b}$. The maximum point wise errors and rate of convergence are presented in Table 5 for this boundary value problem for different values of perturbation parameter $\varepsilon$.

\section{Conclusions}

In this work, a time dependent singularly perturbed differential-difference convection-diffusion equation is solved numerically by using an adaptive grid method. To discretize the domain, we used uniform mesh in the temporal direction and an adaptive mesh has been generated using the concept of entropy function for the spatial di- 


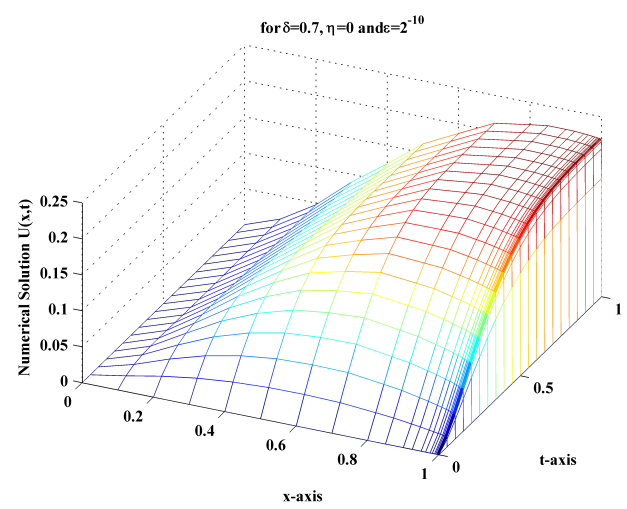

(a) $M^{\star}=26, N=20$

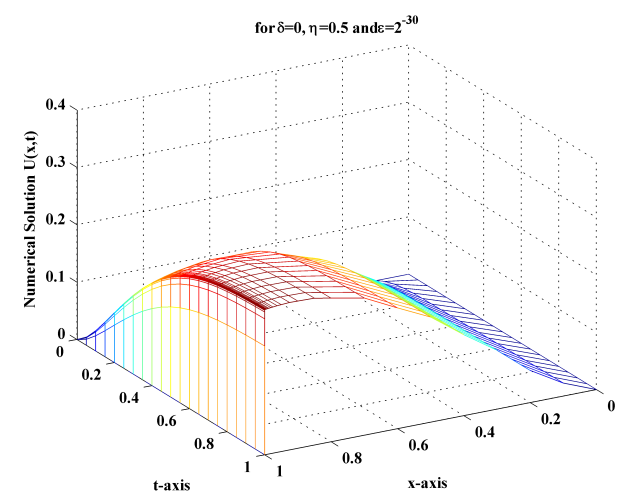

(b) $M^{\star}=68, N=20$

Fig. 6: Surface plot of example 5.2, using adaptive mesh for different values of $\epsilon, \delta$ and $\eta$.

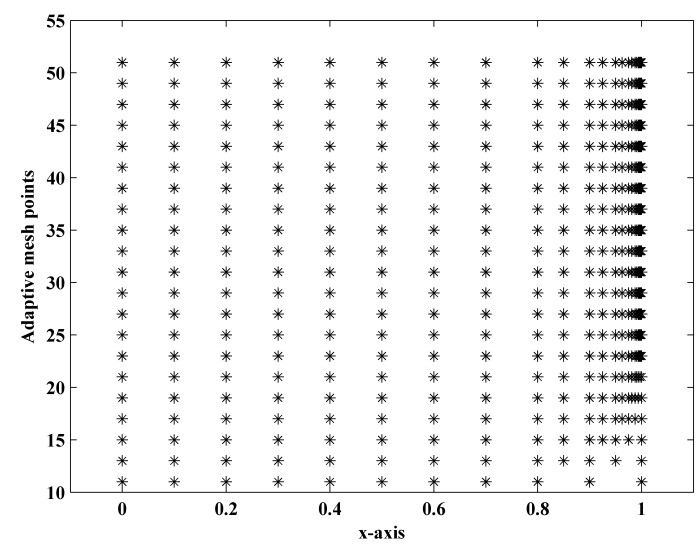

Fig. 7: Adaptive mesh generation for example 5.3 with $\varepsilon=2^{-30}$, $\delta=0.1, \eta=0.2, \mathrm{M}=10$ (initially) and $\mathrm{N}=20$.

rection. The method is based on central finite difference scheme. It has been found that our algorithm gives oscillation free solution with a minimum no of mesh points. Numerical results are carried out to show the efficiency and accuracy of the method. From the results it can be
Table 5: Maximum point wise errors of the solution and corresponding rate of convergence for Example 5.3 by taking $\delta=0.1, \eta=0.2$, $M=10$ and $N=20$ for different values of $\varepsilon$.

\begin{tabular}{cccc}
\hline$\varepsilon$ & $\begin{array}{c}\text { Generated mesh } \\
\left(M^{\star}\right)\end{array}$ & Max. error & $\begin{array}{c}\text { Rate of Convergence } \\
\left(R^{M^{\star}, N}\right)\end{array}$ \\
\hline $2^{-10}$ & 20 & $4.0629 \mathrm{e}-03$ & 0.7271 \\
$2^{-11}$ & 22 & $4.1600 \mathrm{e}-03$ & 0.7421 \\
$2^{-12}$ & 24 & $4.2101 \mathrm{e}-03$ & 0.7483 \\
$2^{-13}$ & 26 & $4.2353 \mathrm{e}-03$ & 0.7514 \\
$2^{-14}$ & 28 & $4.2479 \mathrm{e}-03$ & 0.7533 \\
$2^{-15}$ & 30 & $4.2542 \mathrm{e}-03$ & 0.7544 \\
$2^{-16}$ & 32 & $4.2573 \mathrm{e}-03$ & 0.7551 \\
$2^{-17}$ & 34 & $4.2589 \mathrm{e}-03$ & 0.7555 \\
$2^{-18}$ & 36 & $4.2597 \mathrm{e}-03$ & 0.7557 \\
$2^{-19}$ & 38 & $4.2600 \mathrm{e}-03$ & 0.7558 \\
$2^{-20}$ & 40 & $4.2603 \mathrm{e}-03$ & 0.7558 \\
$2^{-21}$ & 42 & $4.2604 \mathrm{e}-03$ & 0.7559 \\
$2^{-22}$ & 44 & $4.2604 \mathrm{e}-03$ & 0.7559 \\
$2^{-23}$ & 46 & $4.2604 \mathrm{e}-03$ & 0.7559 \\
$2^{-24}$ & 48 & $4.2604 \mathrm{e}-03$ & 0.7559 \\
$2^{-25}$ & 50 & $4.2604 \mathrm{e}-03$ & 0.7559 \\
$2^{-26}$ & 52 & $4.2604 \mathrm{e}-03$ & 0.7559 \\
$2^{-27}$ & 54 & $4.2604 \mathrm{e}-03$ & 0.7559 \\
$2^{-28}$ & 56 & $4.2604 \mathrm{e}-03$ & 0.7559 \\
$2^{-29}$ & 58 & $4.2604 \mathrm{e}-03$ & 0.7559 \\
$2^{-30}$ & 60 & $4.2604 \mathrm{e}-03$ & 0.7559 \\
\hline
\end{tabular}

observed that, the method converges uniformly with respect to the perturbation parameter $\varepsilon$. It is also observed and shown in figures that how perturbation parameter $\varepsilon$ and shift parameters $\delta$ and $\eta$ effect the boundary layer behaviour of solutions. From the numerical results, it is concluded that our adaptive mesh offers a significant advantage with compare to Bakhvalov and Shishkin meshes. The computation is simple and intuitive.

\section{References}

[1] O. Arino, M.L. Hbid, E. Ait Dads, Delay differential equations and applications, Springer, Berlin, 2006.

[2] N.S. Bakhvalov, On the optimization of the methods for solving boundary value problems in the presence of boundary layers, Zh. Vychisl. Mater. Fiz. 9(1969), pp. 841-859.

[3] K. Bansal, K.K. Sharma, Parameter uniform numerical scheme for time dependent singularly perturbed convection-diffusionreaction problems with general shift arguments, Numer. Algor. 75(1)(2017), pp. 113-145.

[4] K. Bansal, P. Rai, K.K. Sharma, Numerical treatment for the class of time dependent singularly perturbed parabolic problems with general shift arguments, Differ. Equ. Dyn. Syst. 25(2)(2017), pp. 327-346.

[5] O. Cheng, M. Jia-qi, The nonlinear singularly perturbed problems for predator-prey reaction diffusion equation, J. 


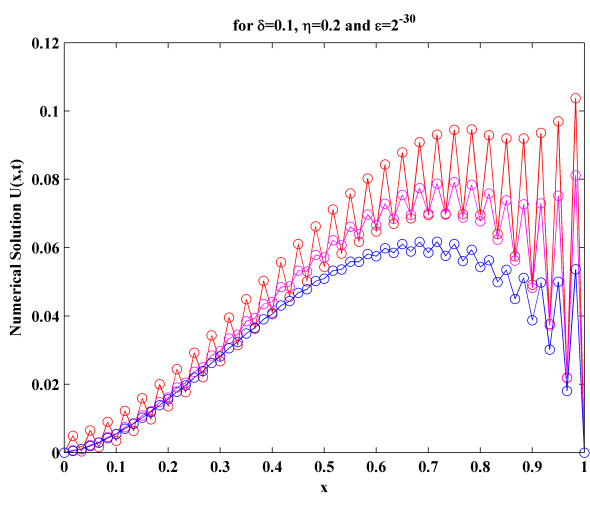

(a) Uniform mesh.

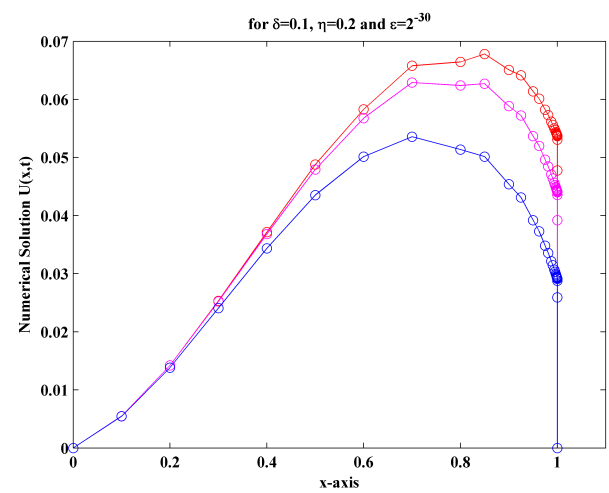

(b) Adaptive mesh.

Fig. 8: Numerical solution of example 5.3, using uniform mesh and adaptive mesh for three different time level with $\varepsilon=2^{-30}, \delta=$ $0.1, \eta=0.2, \mathrm{M}=60$ and $\mathrm{N}=20$.

Biomath. 20(2)(2005), pp. 135-141.

[6] E.R. Doolan, J.J.H. Miller, W.H.A. Schilders, Uniform numerical methods for problems with initial and boundary layers, Boole Press, Dublin, 1980.

[7] A. Friedman, Partial differential equations of parabolic type, Prentice-hall, Englewood Cliffs, 1964.

[8] E.C. Gartland, Graded-mesh difference schemes for singularly perturbed two-point boundary value problems, Math. Comput. 51(184)(1988), pp. 631-657.

[9] D. Kumar, M.K. Kadalbajoo, A parameter-uniform numerical method for time dependent singularly perturbed differentialdifference equations, Appl. Math. Model. 35(6)(2011), pp. 2805-2819.

[10] V. Kumar, B. Srinivasan, An adaptive mesh strategy for singularly perturbed convection diffusion problems, Appl. Math. Model. 39(2015), pp. 2081-2091.

[11] C.G. Lange, R.M. Miura, Singular perturbation analysis of boundary-value problems for differential-difference equations, SIAM J. Appl. Math. 42(1982), pp.502-531.

[12] J.J.H. Miller, E.O. Riordan and I.G. Shishkin, Fitted numerical methods for singular perturbation problems, Word Scientific, Singapore, 1996.

[13] M. Musila, P. Lansky, Generalized Stein's model for anatomically complex neurons, BioSystems. 25(3)(1991), pp. 179-191.

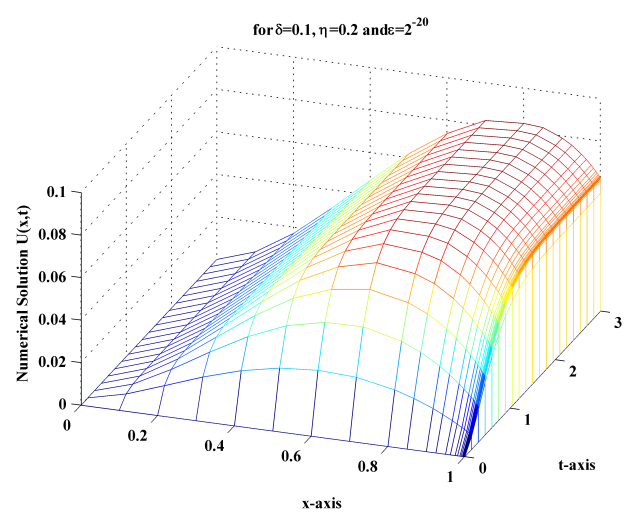

(a) $M^{\star}=40, N=20$

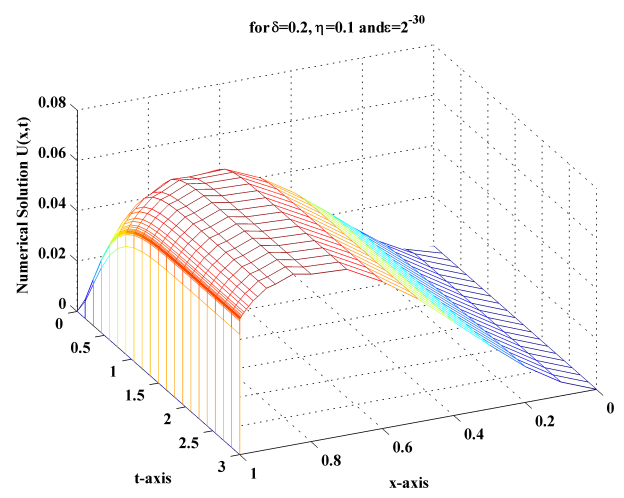

(b) $M^{\star}=60, N=20$

Fig. 9: Surface plot of example 5.3, using adaptive mesh for different values of $\epsilon, \delta$ and $\eta$.

[14] J.D. Murray, Mathematical Biology I: An Introduction, third edition, Springer-Verlag, Berlin, 2001.

[15] V.P. Ramesh, M.K. Kadalbajoo, Upwind and midpoint upwind difference method for time dependent singularly perturbed differential-difference equations with layer behavior, Appl. Math. Comput. 202(2)(2008), pp. 453-471.

[16] R.N. Rao, P.P. Chakravarthy, Fitted numerical methods for singularly perturbed one-dimensional parabolic partial differential equations with small shifts arising in the modelling of neuronal variability, Differ. Equ. Dyn. Syst. (2017), pp. 1-18.

[17] H.G. Roos, M. Stynes and L. Tobiska, Numerical Methods for Singularly Perturbed Differential Equations, Springer-Verlag, Berlin, 2008.

[18] V.A. Solonnikov, O.A. Ladyzenskaja and N.N. Ural'ceva, Linear and quasi linear equations of parabolic type, American Mathematical Society Providence, Rhode Island, 1988.

[19] R.B. Stein, A theoretical analysis of neuronal variability, Biophys.J. 5(2)(1965), pp. 173-194.

[20] J. Wu, Theory and applications of partial functional differential equations, Springer, NewYork, 1996. 